\title{
Prävention und Therapie
}

\section{Gallensteine und GERD: Neues aus den Leitlinien}

Noch in diesem Jahr wird eine aktualisierte Form der S3-Leitlinie aus dem Jahr 2008 zur Diagnostik und Therapie von Gallensteinen erwartet. Für die gastroösophageale Refluxkrankheit (GERD) sind die neuesten Empfehlungen bereits veröffentlicht.

\section{Empfehlung zur Prävention von Gallensteinen}

Erstmals wird in der S3-Leitlinie eine Empfehlung zur Prävention von Cholesteringallenblasensteinen und biliären Symptomen ausgesprochen: Regelmäßige körperliche Aktivität und bedarfsgerechte Ernährung zur Erhaltung eines möglichst normalen Körpergewichts können, wie Prof. Dr. Frank Lammert vom Universitätsklinikum des Saarlandes ausführte, der Entstehung vorbeugen.

\section{Auch asymptomatische \\ Gallengangssteine entfernen}

Um Komplikationen zu verhindern, sollte bei der akuten Cholezystitis frühzeitig, innerhalb von 24 Stunden nach stationärer Aufnahme, laparoskopisch cholezystektomiert werden.

Während symptomatische Gallengangssteine schon immer als behandlungsbedürftig galten, sahen dies die Empfehlungen für asymptomatische Gallengangssteine bislang nicht vor: Einerseits gab es zu wenige Daten über den natürlichen Verlauf asymptomatischer Gallengangssteine, andererseits war bekannt, dass mehr als $20 \%$ der Steine spontan abgehen und weniger als 50\% symptomatisch werden.

Eine retrospektive Analyse des schwedischen GallRisk-Registers mit 3828 Patienten brachte jetzt neue Daten ans Licht: Demnach liegt die Komplikationsrate (Kolik, Cholangitis, Pankreatitis) bei Patienten, deren asymptomatische Gallengangssteine nicht entfernt werden, bei 25\%, nach operativer Entfernung jedoch

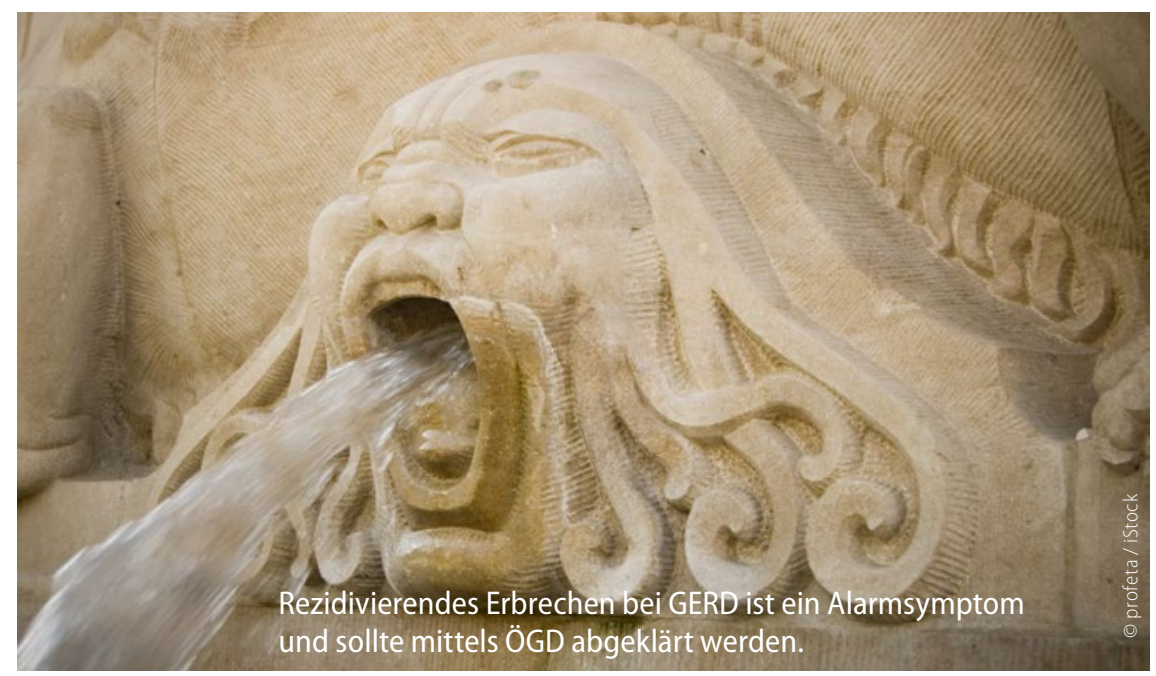

nur bei 13\%. Ähnliche Zahlen ergaben sich, wenn kleine $(<4 \mathrm{~mm})$ und mittlere (4-8 $\mathrm{mm}$ ) Steine separat analysiert wurden [1]. Die neue Leitlinie sieht deshalb vor, auch asymptomatische Gallengangssteine zu behandeln.

\section{Simultane Choledocho- und Cholezystolithiasis}

Sind Gallenblase und Gallengänge gleichzeitig von Steinen betroffen, sollte die Therapie gesplittet werden: Nach einer erfolgreichen endoskopischen oder perkutanen Gallengangssanierung muss sich möglichst innerhalb von 72 Stunden eine Cholezystektomie anschließen. Dies senkt die Raten an biliären Koliken und akuter Cholezystitis auf 0 , während diese bei späterer Cholezystektomie nach sechs bis acht Wochen deutlich ansteigen.

\section{Gastroösophageale \\ Refluxkrankheit (GERD)}

Noch immer, so das Fazit von Prof. Dr. Herbert Koop aus Berlin zur aktualisierten s2k-Leitlinie der GERD, stelle die Anamnese den wichtigsten diagnostischen Baustein für die Refluxkrankheit dar. Nicht zwangsläufig muss demnach bei Erwachsenen, wenn sie unter typi- schen Refluxsymptomen leiden, eine umfassende Diagnostik erfolgen: Wird eine GERD angenommen und liegen keine Alarmsymptome vor, kann eine empirische Therapie mit Protonenpumpeninhibitoren (PPI) erfolgen. Sind dagegen Alarmsymptome wie Dysphagie, Odynophagie, rezidivierendes Erbrechen, (unfreiwillige) Gewichtsabnahme, Anämie, Hinweise auf einen GI-Blutverlust oder eine Raumforderung vorhanden, sollte eine Ösophago-Gastro-Duodenoskopie (ÖGD) durchgeführt werden.

Sprechen Refluxsymptome auf eine empirische PPI-Therapie nicht an, soll zur Abklärung bevorzugt eine 24-Stunden-pH-Metrie-Mll (multikanale intraluminale Impedanzmessung) angewendet werden. In der Praxis, so Koop, bedeute dies eine hohe Hürde, weil das Verfahren bei potenziell hohen Patientenzahlen nur beschränkt verfügbar sei.

(Kathrin von Kieseritzky)

[1] Möller M et al. Natural Course vs Interventions to Clear Common Bile Duct Stones: Data From the Swedish Registry for Gallstone Surgery and Endoscopic Retrograde Cholangiopancreatography (GallRiks). JAMA Surg 2014; 149(10):1008-1013;

Viszeralmedizin 2015, 16-19. September 2015 in Leipzig, Session: DGVS-Leitlinien - Was ist neu? 